\title{
Chromospheric activity on the RS CVn-type binary UX Arietis
}

\author{
S.-h. Gu, H.-s. Tan, H.-g. Shan, and F.-h. Zhang
}

\author{
Yunnan Observatory, Joint laboratory for optical astronomy, National Astronomical Observatories, \\ Chinese Academy of Sciences, Kunming, PR China \\ e-mail: hgshan@public.km.yn.cn
}

Received 20 December 2001 / Accepted 19 March 2002

\begin{abstract}
High resolution spectroscopic observations of UX Ari made during three observing seasons in 2000 have been analyzed by means of the spectral subtraction technique. The information about chromospheric activity of UX Ari has been obtained from several optical chromospheric activity indicators such as the HeI $\mathrm{D}_{3}$, NaI $\mathrm{D}_{1}$, $\mathrm{D}_{2}, \mathrm{H}_{\alpha}$, and CaII IRT lines. The results suggest the chromospheric activity of the system is associated with the cooler secondary. An orbital phase modulation of chromospheric emissions is found, and consequently the activity longitude area of the system is obtained, that corresponds to the phase interval near the second quadrature of the binary system. A flare-like event is detected during our new observations, characterized by the dramatic increase in the $\mathrm{H}_{\alpha}$ and CaII IRT emission lines, that is confirmed by the emission of the HeI $\mathrm{D}_{3}$ line and the filled-in cores of the NaI $D_{1}, D_{2}$ lines. It is found that the values of the $E W_{8542} / E W_{8498}$ ratio decrease when the activity of the system increases, especially when the flare occurs. The small values of the $E W_{8542} / E W_{8498}$ ratio indicate that the CaII IRT emissions come from plage-like regions. The high activity level of UX Ari around the quadrature may originate with the coupling between the chromospheric activity of the secondary and the mass transfer activity of the two components.
\end{abstract}

Key words. stars: activity - stars: binaries: close - stars: chromosphere - stars: flare - stars: individual: UX Ari

\section{Introduction}

UX Ari is one of the most active RS CVn binaries that always show $\mathrm{H}_{\alpha}$ emission above continuum. It is a noneclipsing double-lined spectroscopic binary with spectral type G5V/K0IV (Carlos \& Popper 1971) and orbital period 6. 4378553 (Duemmler \& Aarum 2001). The observations of several chromospheric activity indicators (Frasca \& Catalano 1994; Huenemoerder et al. 1989; Montes et al. 1995; Montes et al. 1996; Montes et al. 2000; Nations \& Ramsey 1986) have demonstrated that the chromospheric activity of UX Ari is very strong in the group of RS CVn binaries and the chromospheric activity of this system is associated with the cooler secondary component.

Recently, the spectral subtraction technique has been used widely for chromospherically active binaries in several lines CaII H\&K, MgIb, HeI $\mathrm{D}_{3}$, NaI $\mathrm{D}_{1}, \mathrm{D}_{2}, \mathrm{H}_{\alpha}, \mathrm{H}_{\beta}$, CaII IRT (Gunn \& Doyle 1997; Gunn et al. 1997; Lázaro \& Arévalo 1997; Montes et al. 1997; Montes et al. 2000 etc.). By using this method, more and more information about chromospheric activity on stars has become known. This is very important for us to well understand the behaviour of chromospherically active binaries.

Send offprint requests to: S.-h. Gu, e-mail: hgshan@public.km.yn.cn
Here new spectroscopic observations of UX Ari are analyzed by using the spectral subtraction technique, and the excess emissions in several chromospheric activity indicators are determined. Based on these observations and analyses, some results are derived for the chromospheric activity of UX Ari. In Sect. 2, we give details about our observations and data reduction. In Sect. 3, we describe the procedure of spectral analysis. In Sect. 4 , the behaviours of different chromospheric activity indicators are reported. Finally, the discussion and conclusions are given in Sect. 5 .

\section{Observation and data reduction}

The spectroscopic observations of UX Ari were carried out with the $2.16 \mathrm{~m}$ telescope at the Xinglong station of the National Astronomical Observatories, China during three observing seasons: February 19-24, September 15-17, and November 11 2000. The Coude echelle spectrograph with a spectral resolution about 34000 and a $1024 \times 1024$ pixel $\mathrm{CCD}$ detector was used during the observations. The reciprocal dispersions are $0.082 \AA /$ pixel for the HeI $\mathrm{D}_{3}$ and NaI $D_{1}, D_{2}$ line spectral region, $0.091 \AA /$ pixel for the $\mathrm{H} \alpha$ line spectral region, $0.119 \AA$ /pixel for the CaII IRT $(8498.06 \AA, 8542.14 \AA)$ line spectral region, and $0.120 \AA /$ pixel for the CaII IRT (8662.17 $\AA$ ) line spectral region. Correspondingly, the spectral resolution determined 
Table 1. The observational log of UX Ari.

\begin{tabular}{llll}
\hline \hline Date & UT & Phase & Exposure time (s) \\
\hline 2000.02 .19 & $13: 10: 06$ & 0.132 & 1200 \\
2000.02 .19 & $13: 32: 19$ & 0.135 & 1200 \\
2000.02 .20 & $14: 13: 40$ & 0.295 & 1200 \\
2000.02 .20 & $14: 34: 50$ & 0.297 & 1200 \\
2000.02 .21 & $14: 41: 11$ & 0.453 & 3600 \\
2000.02 .22 & $13: 20: 54$ & 0.600 & 1200 \\
2000.02 .22 & $13: 41: 31$ & 0.602 & 1200 \\
2000.02 .23 & $13: 21: 30$ & 0.755 & 1800 \\
2000.02 .23 & $13: 52: 19$ & 0.758 & 1800 \\
2000.02 .24 & $13: 07: 26$ & 0.909 & 1200 \\
2000.02 .24 & $13: 28: 20$ & 0.911 & 1200 \\
2000.09 .15 & $19: 23: 13$ & 0.625 & 1800 \\
2000.09 .16 & $17: 28: 18$ & 0.781 & 1800 \\
2000.09 .17 & $17: 32: 30$ & 0.933 & 1800 \\
2000.11 .11 & $17: 01: 55$ & 0.492 & 1200 \\
\hline
\end{tabular}

as the $F W H M$ of the arc comparison lines is $0.151,0.167$, $0.212,0.215 \AA$, respectively. The signal to noise ratio $(S / N)$ is more than 100 for all observations. The observing $\log$ is listed in Table 1. For each observation, the observing date, UT, orbital phase, and exposure time are given.

The spectral images are reduced by means of the IRAF package. The standard procedures are used, which include image trimming, bias subtraction, flat-field division, and 1-D spectrum extraction. The wavelength calibration was obtained by taking the spectrum of a Th-Ar lamp. Finally, all spectra have been normalized by a spline 3 fitting to the continuum of the observed spectra.

On some nights of our observing seasons when the telluric lines were heavy, two rapidly rotating early-type stars HR8858 (B5V, $v \sin i=332 \mathrm{~km} \mathrm{~s}^{-1}$ ) and HR7894 (B5IV, $\left.v \sin i=330 \mathrm{~km} \mathrm{~s}^{-1}\right)$ were observed. Their observed spectra are fitted by using high order spline3 function to derive the telluric line templates. The telluric lines in the spectra of UX Ari are removed by means of these templates. Figure 1 shows examples of such a procedure in different spectral regions.

\section{Spectral analysis}

The normalized spectra of UX Ari are analyzed using the spectral subtraction technique described in detail by Barden (1985) and Montes et al. (1995). In this method, the synthesized spectrum is constructed from artificially rotationally broadened, radial-velocity shifted, and weighted spectra of two inactive stars with the same spectral-type and luminosity class as the two components of the active star. For our situation, the synthesized spectrum is constructed by means of the program STARMOD (Barden 1985); the two inactive stars HR 3309 (G5V) and HR 3351 (K0IV) observed in the same campaign are used as reference stars.

In the course of the analysis, the $v \sin i$ values $\left(7.5 / 39 \mathrm{~km} \mathrm{~s}^{-1}\right)$ of the two components of UX Ari were
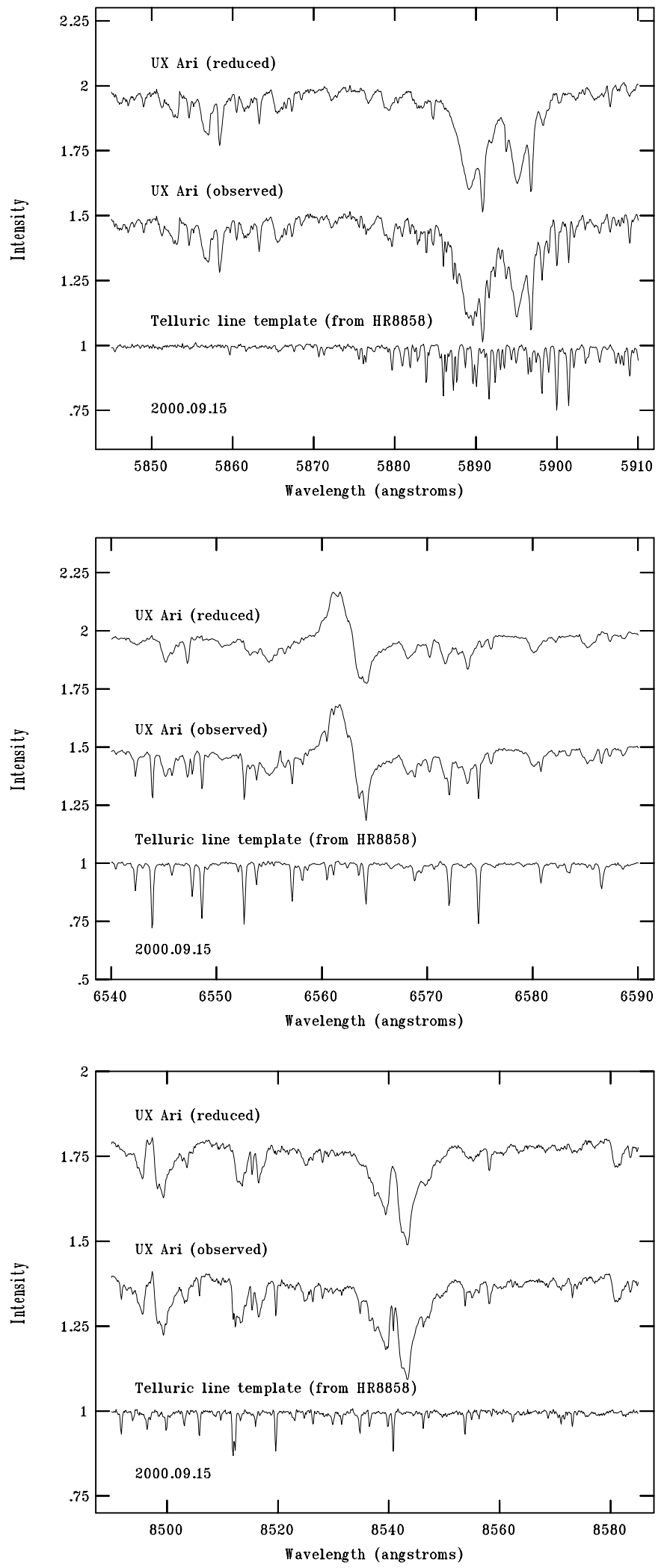

Fig. 1. The illustrations for removing telluric lines from the observed spectra in HeI $\mathrm{D}_{3}$, NaI $\mathrm{D}_{1}, \mathrm{D}_{2}, \mathrm{H}_{\alpha}$, and CaII IRT wavelength bands.

taken from Duemmler \& Aarum (2001), the intensity weight for each of the two components was obtained from 
Table 2. The measurements for the excess emissions of the $\mathrm{H} \alpha$ and CaII IRT lines.

\begin{tabular}{|c|c|c|c|c|c|}
\hline Phase & $F W H M(\AA)$ & $E W(\AA)$ & $I_{\mathrm{c}}$ & $\log F_{\mathrm{S}}\left(\mathrm{erg} \mathrm{cm}^{-2} \mathrm{~s}^{-1}\right)$ & $E W_{8542} / E W_{8498}$ \\
\hline & $\mathrm{H} \alpha$, CaII $(8498,8542,8662)$ & $\mathrm{H} \alpha$, CaII $(8498,8542,8662)$ & $\mathrm{H} \alpha$, CaII $(8498,8542,8662)$ & $\mathrm{H} \alpha$, CaII $(8498,8542,8662)$ & \\
\hline 0.132 & 1.8591 .6941 .9681 .929 & $\begin{array}{llll}1.365 & 0.600 & 0.973 & 0.816\end{array}$ & $\begin{array}{lllll}0.656 & 0.318 & 0.407 & 0.374\end{array}$ & 6.8156 .4006 .6106 .523 & 1.621 \\
\hline 0.135 & $\begin{array}{llll}1.871 & 1.702 & 1.971 & 1.916\end{array}$ & $\begin{array}{llll}1.364 & 0.591 & 0.943 & 0.793\end{array}$ & $\begin{array}{llll}0.670 & 0.309 & 0.403 & 0.356\end{array}$ & 6.8156 .3946 .5966 .510 & 1.594 \\
\hline 0.295 & $\begin{array}{llll}1.922 & 1.698 & 1.970 & 1.933\end{array}$ & $\begin{array}{llll}1.503 & 0.615 & 0.978 & 0.810\end{array}$ & $\begin{array}{llll}0.675 & 0.320 & 0.424 & 0.362\end{array}$ & 6.8576 .4116 .6126 .519 & 1.590 \\
\hline 0.297 & 1.9231 .6911 .9571 .927 & $\begin{array}{llll}1.514 & 0.616 & 0.951 & 0.830\end{array}$ & $\begin{array}{llll}0.685 & 0.327 & 0.417 & 0.367\end{array}$ & 6.8606 .4126 .6006 .530 & 1.543 \\
\hline 0.453 & $2.0971 .7341 .977 \quad 1.977$ & $\begin{array}{llll}1.370 & 0.628 & 0.922 & 0.779\end{array}$ & $\begin{array}{llll}0.581 & 0.311 & 0.404 & 0.351\end{array}$ & 6.8176 .4206 .5876 .502 & 1.467 \\
\hline 0.600 & 1.8081 .6141 .9021 .848 & $\begin{array}{llll}1.363 & 0.594 & 0.934 & 0.836\end{array}$ & $\begin{array}{llll}0.678 & 0.355 & 0.421 & 0.401\end{array}$ & $6.8156 .396 \quad 6.5926 .533$ & 1.572 \\
\hline 0.602 & $\begin{array}{llll}1.808 & 1.630 & 1.895 & 1.874\end{array}$ & $\begin{array}{llll}1.373 & 0.598 & 0.903 & 0.807\end{array}$ & $\begin{array}{llll}0.693 & 0.343 & 0.405 & 0.372\end{array}$ & 6.8186 .3986 .5786 .518 & 1.510 \\
\hline 0.755 & $\begin{array}{llll}1.840 & 1.669 & 1.897 & 1.976\end{array}$ & $\begin{array}{llll}1.716 & 0.650 & 0.972 & 0.906\end{array}$ & $\begin{array}{llll}0.830 & 0.370 & 0.451 & 0.402\end{array}$ & 6.9156 .4356 .6106 .568 & 1.495 \\
\hline 0.758 & 1.8131 .6651 .9411 .958 & $\begin{array}{llll}1.713 & 0.639 & 0.949 & 0.893\end{array}$ & $\begin{array}{llll}0.828 & 0.360 & 0.434 & 0.374\end{array}$ & 6.9146 .4276 .5996 .562 & 1.486 \\
\hline 0.909 & 2.0651 .6951 .9872 .032 & $\begin{array}{llll}1.593 & 0.576 & 0.851 & 0.792\end{array}$ & $\begin{array}{llll}0.723 & 0.323 & 0.384 & 0.340\end{array}$ & 6.8826 .3836 .5526 .510 & 1.477 \\
\hline 0.911 & $2.062 \quad 1.708 \quad 1.9862 .010$ & $\begin{array}{llll}1.578 & 0.576 & 0.845 & 0.800\end{array}$ & $\begin{array}{llll}0.719 & 0.314 & 0.384 & 0.339\end{array}$ & 6.8786 .3836 .5496 .514 & 1.467 \\
\hline 0.492 & 1.9601 .6431 .9191 .935 & $\begin{array}{llll}1.421 & 0.653 & 1.111 & 0.937\end{array}$ & $\begin{array}{lllll}0.653 & 0.354 & 0.469 & 0.400\end{array}$ & 6.8336 .4376 .6686 .582 & 1.701 \\
\hline 0.625 & $1.958 \quad 1.647 \quad 1.8851 .941$ & $\begin{array}{llll}1.481 & 0.614 & 0.953 & 0.821\end{array}$ & $\begin{array}{llll}0.683 & 0.323 & 0.425 & 0.354\end{array}$ & 6.8516 .4106 .6016 .525 & 1.552 \\
\hline 0.781 & $\begin{array}{llll}1.911 & 1.589 & 1.683 & 1.791\end{array}$ & $\begin{array}{llll}2.155 & 0.783 & 0.951 & 0.986\end{array}$ & $\begin{array}{llll}0.992 & 0.468 & 0.539 & 0.488\end{array}$ & 7.0136 .5166 .6006 .605 & 1.214 \\
\hline 0.933 & $2.111 \quad 1.917 \quad 1.9502 .019$ & $\begin{array}{llll}1.870 & 0.742 & 0.818 & 0.813\end{array}$ & $\begin{array}{llll}0.804 & 0.323 & 0.381 & 0.344\end{array}$ & 6.9526 .4936 .5356 .521 & 1.102 \\
\hline
\end{tabular}

two spectra taking at phases 0.295 and 0.297 , where the two components are well separated. Finally, the adopted intensity weight ratios are $0.30 / 0.70$ for HeI $\mathrm{D}_{3}$ and NaI $\mathrm{D}_{1}, \mathrm{D}_{2}$ line spectral region, $0.24 / 0.76$ for $\mathrm{H} \alpha$ line spectral region, $0.23 / 0.77$ for CaII IRT (8498.06 $\AA, 8542.14 \AA)$ line spectral region, $0.21 / 0.79$ for CaII IRT (8662.17 $\AA$ ) line spectral region.

The synthesized and subtracted spectra we have obtained in the different spectral regions are displayed in Figs. 2-7, where the orbital phases calculated using Duemmler \& Aarum's (2001) new ephemeris are also marked. In these figures, "H" and "C" represent the primary (hotter component) and the secondary (cooler component), respectively. For the $\mathrm{H} \alpha$ lines and CaII IRT lines, offsets are added to the subtracted spectra for better display.

\section{Chromospheric activity indicators}

The several chromospheric activity indicators in our observations are formed at different heights in the chromosphere, NaI $D_{1}, D_{2}$ are formed in the upper photosphere and lower chromosphere, CaII IRT lines in the lower chromosphere, $\mathrm{H}_{\alpha}$ in the middle chromosphere, and HeI $\mathrm{D}_{3}$ in the upper chromosphere. Through detailed investigation, some information about the chromosphere structure can be inferred.

In all of the activity indicators analysed in our observations, we have found that the emission features in the spectrum come from the cooler component. The main spectroscopic features of the excess emissions in the $\mathrm{H} \alpha$ and CaII IRT lines have been measured in the subtracted spectra and listed in Table 2, which includes orbital phase, full width at half maximum $F W H M$ of the excess emissions, equivalent width $E W$ of the excess emissions, the intensity of the excess emission peak, and the absolute flux (logarithm) at the stellar surface $\log F_{\mathrm{S}}$ derived using the calibration of Hall (1996) as the relation of color in$\operatorname{dex}(V-R)$. The equivalent width $E W \mathrm{~s}$ of the excess emissions are corrected for the contribution of the cooler component to the total continuum before they are converted to the absolute flux $\log F_{\mathrm{S}}$ at the stellar surface. The measured excess emission $E W$ s have been plotted vs. the orbital phase in Fig. 8.

In order to analyse the short time-scale chromospheric variability of UX Ari, we have divided our three observing campaigns into two different periods: February 2000 when the observation almost covers a complete orbital period, and September-November 2000 when the observation covers about half the orbital period. Figures $2-8$ show that HeI $\mathrm{D}_{3}, \mathrm{NaI} \mathrm{D}_{1}, \mathrm{D}_{2}, \mathrm{H} \alpha$, and CaII IRT indicators except the CaII IRT (8542) line during the second period exhibit similar variation patterns in the two periods, and their behaviours are coincident with each other. In the second period, the spectra at orbital phase 0.781 show stronger excess emission in all of the activity indicators. The $\mathrm{H} \alpha$ and CaII IRT emission lines show a dramatic increase, the HeI $D_{3}$ line changes to emission, and the NaI $D_{1}, D_{2}$ lines show a stronger fill-in. According to Zirin (1988), if the HeI D3 line is in emission, it suggests a flare-like event has happened. Therefore, these results suggest that a flare-like event was detected.

When this flare happened, the excess emission $E W$ of $\mathrm{H} \alpha$ line increased by about a factor of 1.5 in one day. This factor is smaller than in the previous flare of UX Ari detected by Montes et al. (1996) during which the excess emission $E W$ of the $\mathrm{H} \alpha$ line increased by about a factor 2.2 in one day. Compared with the typical increase factor of 2 of the chromospheric lines during flares, the flare detected here is somewhat weak. Converting the excess emission $E W$ of the $\mathrm{H} \alpha$ line into luminosity with the radius of the cooler component given by Duemmler \& Aarum (2001), we have derived the energy released in the flare, $2.1 \times 10^{31} \mathrm{erg} \mathrm{s}^{-1}$, which is of the same order as the $\mathrm{H} \alpha$ 

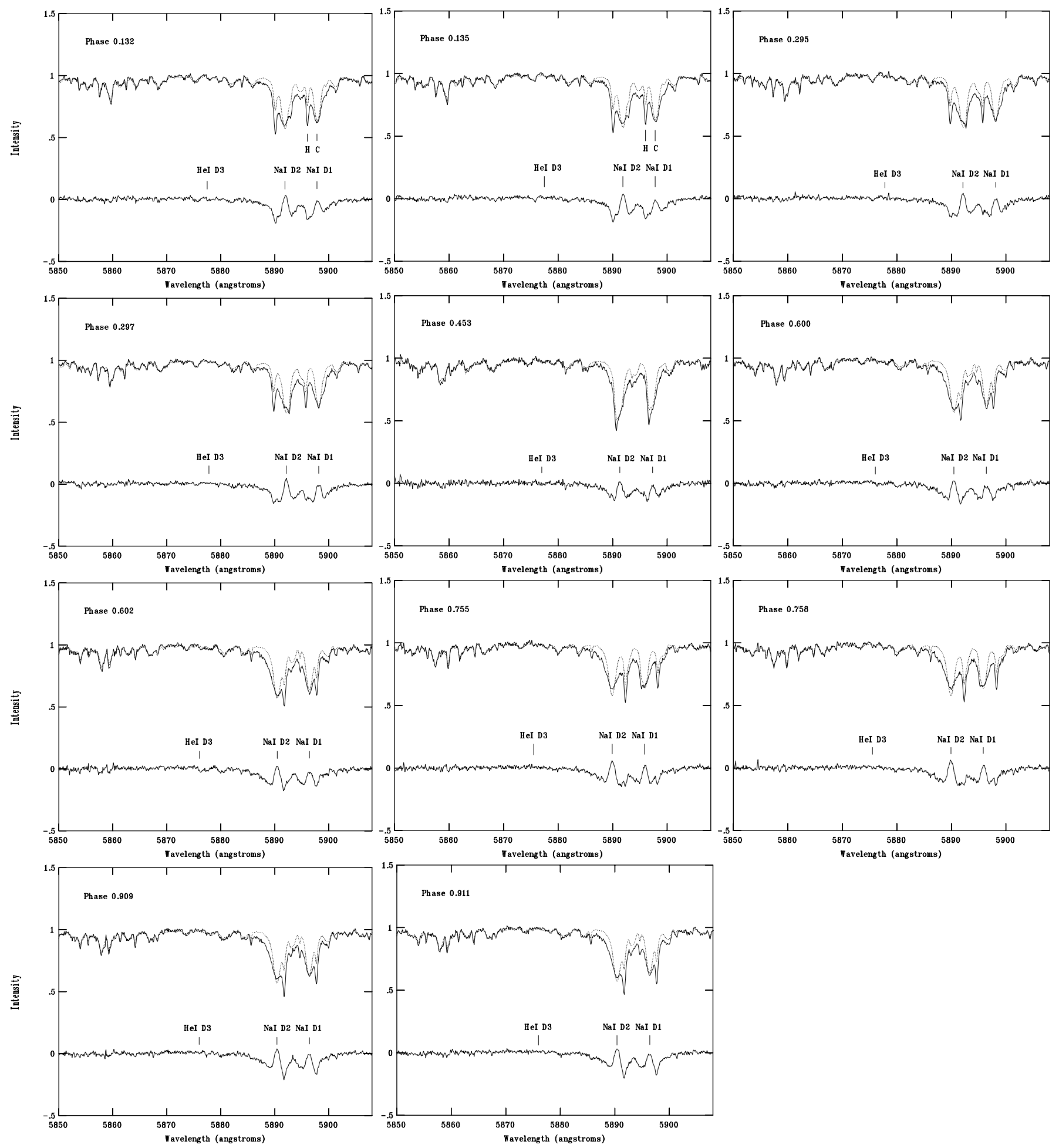

Fig. 2. The profiles of the NaI $D_{1}, D_{2}$, and HeI $D_{3}$ lines in Feb. 2000. The dotted lines represent the synthesized spectra. The lower spectra are the subtracted spectra.

flares in other RS-CVn systems such as HK Lac (Catalano \& Frasca 1994) and HR 1099 (Foing et al. 1994). In addition, the flare we detected took place at the orbital phase 0.781 , which is very similar to the orbital phase (0.74) of the flare detected by Montes et al. (1996), and also to the orbital phase where the excess emission $E W$ of $\mathrm{H} \alpha$ line reached a maximum in our Feb. 2000 observations.
Therefore, the activity longitude region of UX Ari should lie around here.

\section{Discussion and conclusions}

From Figs. 2-7, it is found that the synthesized spectra can match the observational ones, except for the NaI $\mathrm{D}_{1}$, 

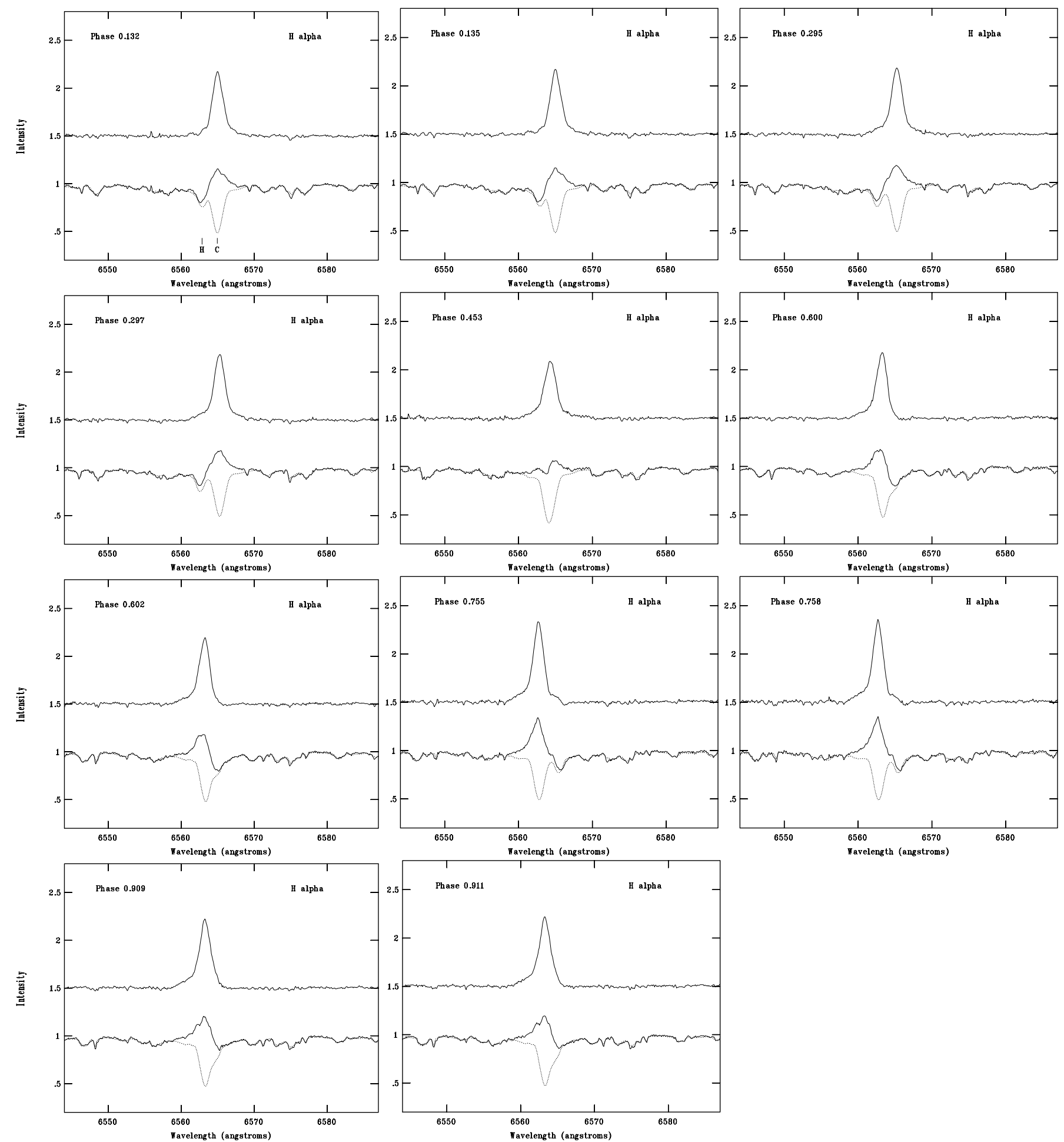

Fig. 3. The profiles of the $H_{\alpha}$ lines in Feb. 2000. The dotted lines represent the synthesized spectra. The upper spectra are the subtracted spectra.

$\mathrm{D}_{2}$ lines, quite well. The reason for mismatched NaI $\mathrm{D}_{1}, \mathrm{D}_{2}$ lines has already been discussed by Montes et al. (1997), and they had concluded that the filled-in core of the NaI $\mathrm{D}_{1}, \mathrm{D}_{2}$ lines could be used as a chromospheric activity indicator. In the subtracted spectra, $\mathrm{H} \alpha$ line emission profiles exhibit broad wings that can arise from microflaring (Montes et al. 1998). They result from the large-scale mass motions in the chromosphere, as in another RS CVn-type binary, DM UMa (Hatzes 1995).

In the course of spectral analysis, it was found that the primary's $\mathrm{H}_{\alpha}$ profiles are deeper during orbital phase $0.60-0.93$ than those during orbital phase $0.13-0.49$. These features mean that the excess absorption of the primary's hydrogen lines mentioned by Huenemoerder et al. (1989) 

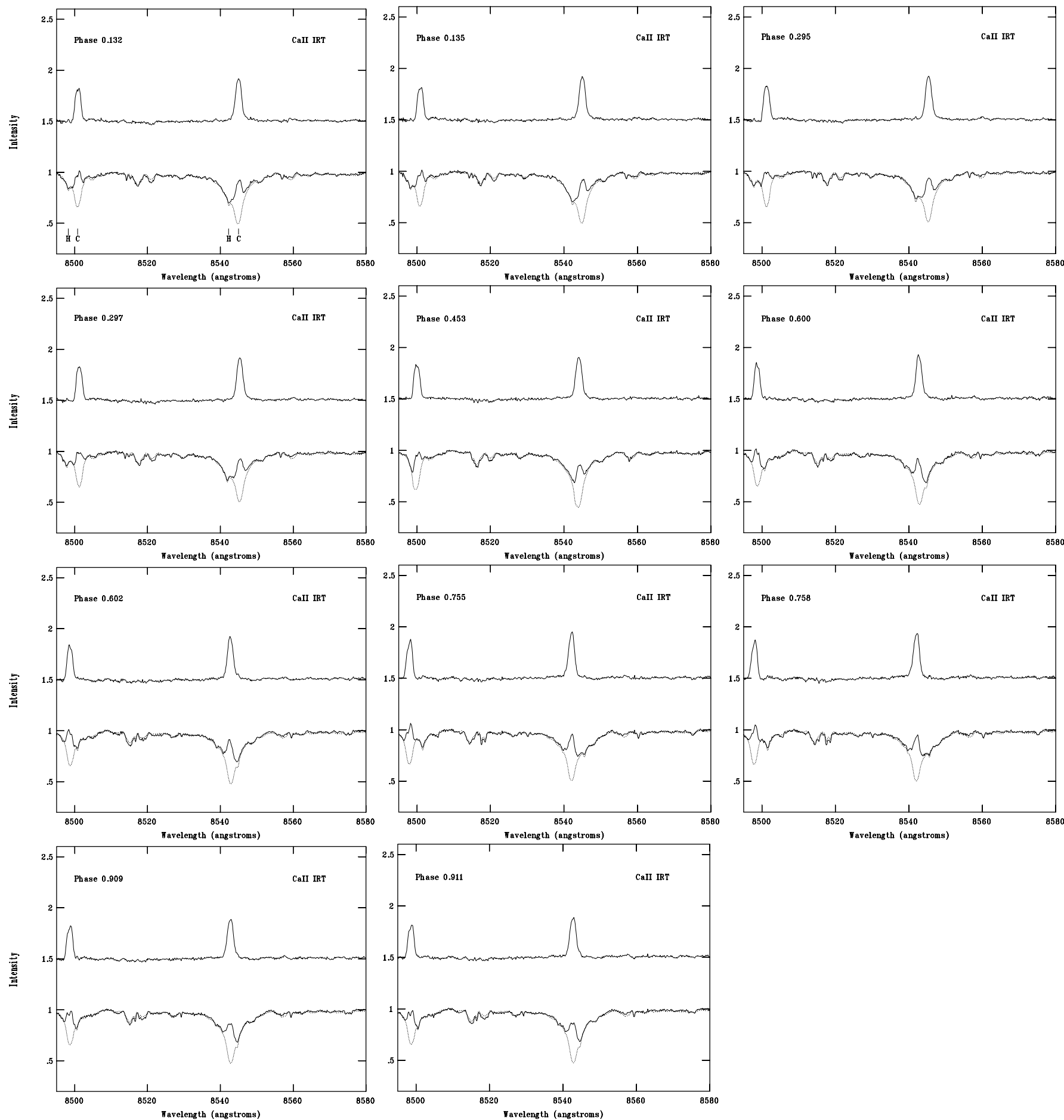

Fig. 4. As Fig. 3, but for the CaII IRT $(8498,8542)$ lines.

also exists in our observations, although these features are not as clear as those in the spectra of Huenemoerder et al. (1989). In addition, Montes et al.'s (1995) spectrum at phase 0.93 also supports this point. Huenemoerder et al. (1989) suggested that the secondary transfers mass to the primary, and these $\mathrm{H}_{\alpha}$ excess absorptions are either caused by a cloud of material ejected by the impact or by the occultation of the mass stream. Although the absolute parameters of UX Ari derived by Duemmler \& Aarum (2001) have demonstrated that the radius of the cooler component is less than its Roche lobe, Huenemoerder et al.'s (1989) interpretation to this feature is still convincing. For the component star with a very active chromosphere and corona, like the secondary of UX Ari, it is very possible that some extended circumstellar material exists that can easily extend beyond the Roche radius and cause the Roche lobe overflow. 

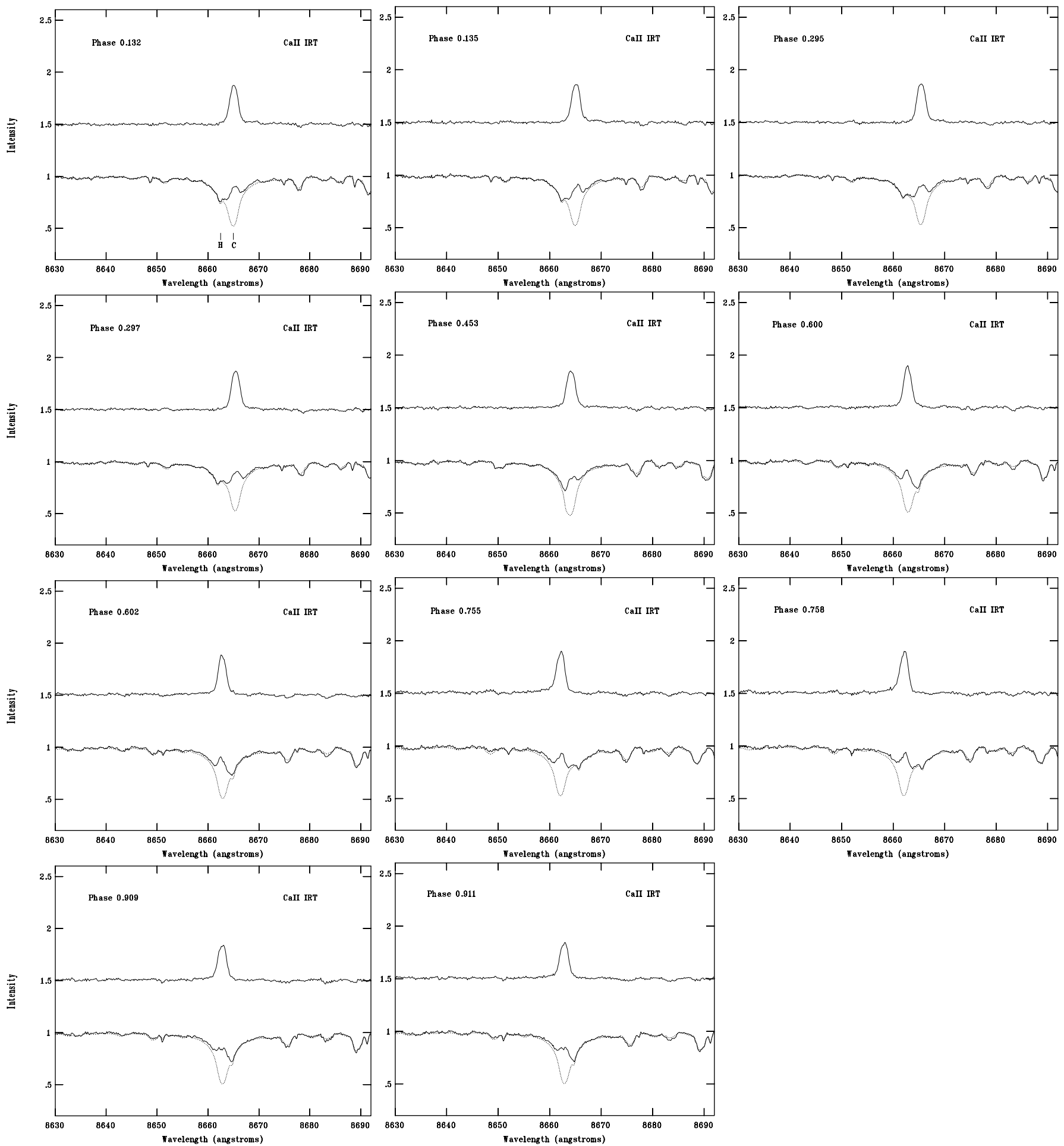

Fig. 5. As Fig. 3, but for the CaII IRT (8662) lines.

In Table 2 and Fig. 8, it can be seen that during the two periods covered by our observations, the level of chromospheric activity is higher around the second quadrature of the binary system (phase 0.75 ) than at the other orbital phases. This indicates that the chromospheric activity is modulated with orbital phase and the inferred activity longitude area of UX Ari corresponds to the phase interval near the second quadrature of the system. This also is the position where there is excess absorption in the primary's hydrogen lines. The high activity level of UX Ari at this phase interval may be the consequence of the coupling between chromospheric activity and mass transfer activity. Because the Roche lobe overflow is a long-lived behavior, it can be the basis of corotating flux tubes contecting the two components. This makes it easier to create higherlevel chromospheric activity. The flare-like event detected 

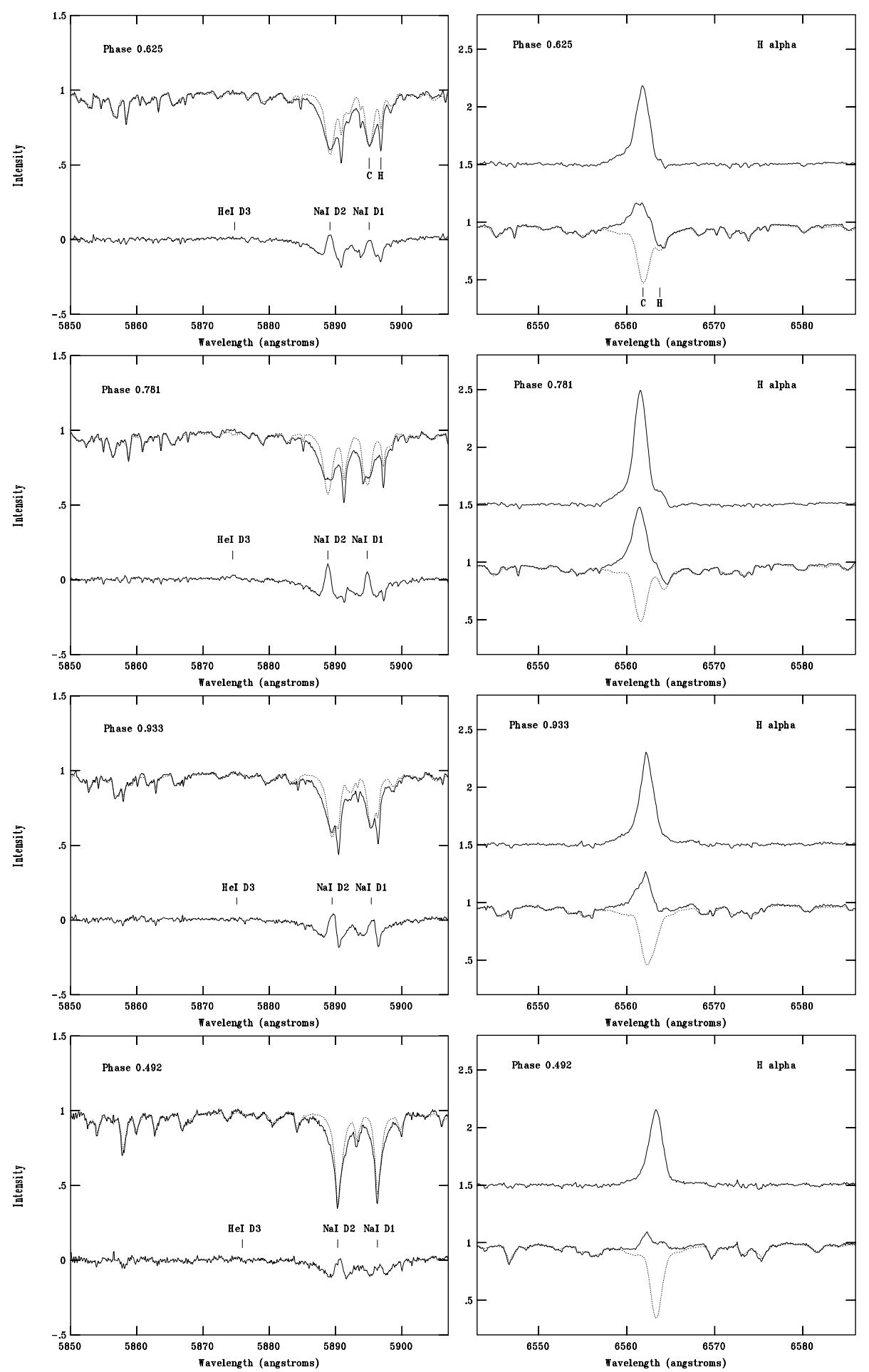

Fig. 6. As Figs. 2 and 3, but for the observations in Sep. \& Nov. 2000.

here is some weaker than the one detected by Montes et al. (1996).

Among all chromospheric activity indicators, the CaII IRT (8542) line exhibits different behaviour to others in the second observational campaign, namely during the flare. From the $E W_{8542} / E W_{8498}$ ratio of CaII IRT (8542 $\AA$ ) and CaII IRT (8498 $\AA$ ) excess emissions, also listed in Table 2, it can be found that the values of the $E W_{8542} / E W_{8498}$ ratio are generally small, as for other active stars (Arévalo \& Lázaro 1999; Montes et al. 2000), and much smaller during the phase interval of high activity and the flare (see Fig. 9). This indicates that CaII IRT emissions arise from the plage-like regions, and the $E W_{8542} / E W_{8498}$ ratio decreases with the increase of chromospheric activity.

Finally, we give a summary in the present study of UX Ari. The activity information is obtained from several optical spectroscopic features. The chromospheric 

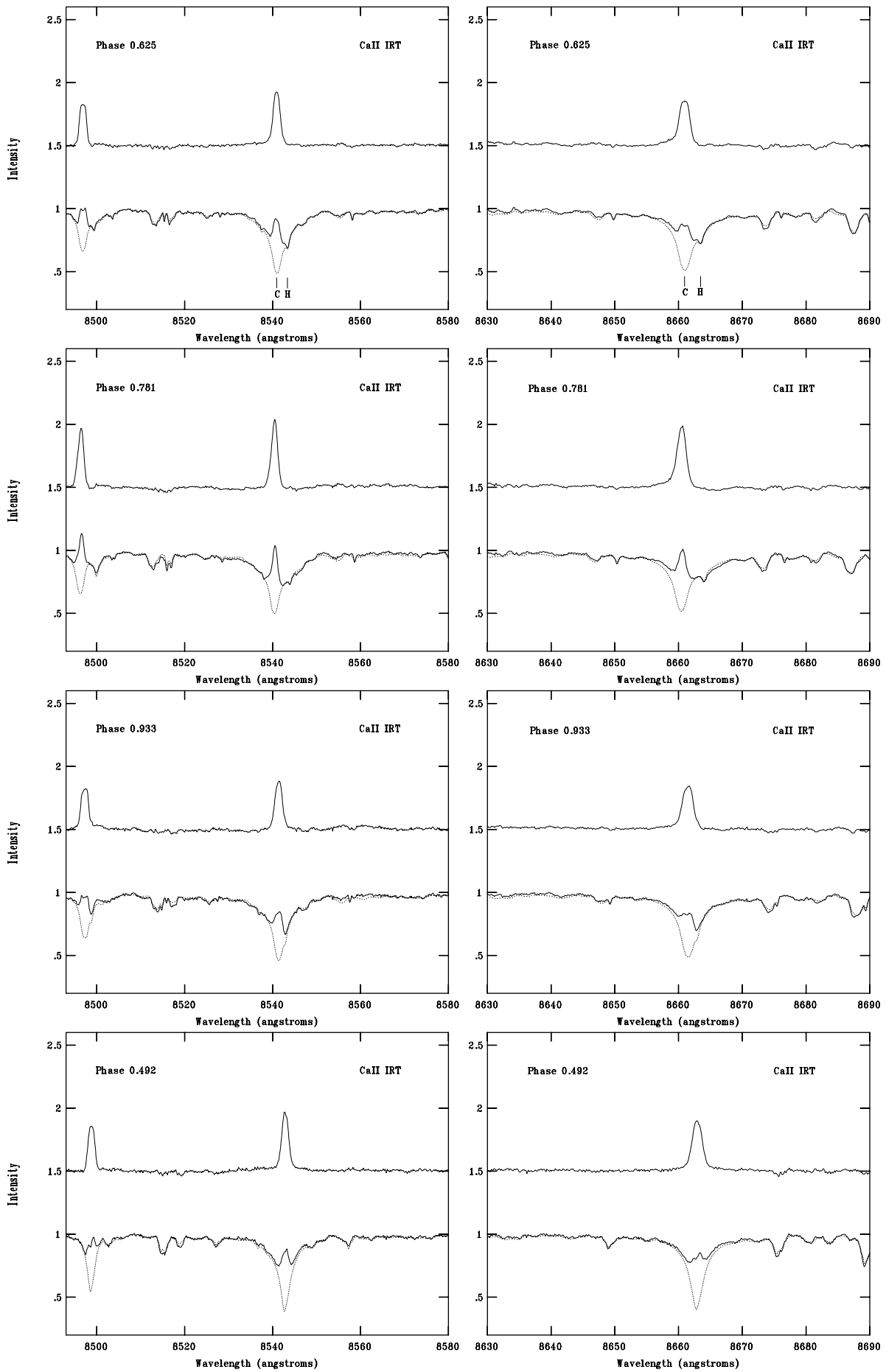

Fig. 7. As Figs. 4 and 5, but for the observations in Sep. \& Nov. 2000.

contribution to these chromospheric activity indicators is determined by the spectral subtraction technique. A flare is detected in our observations, and it is the second time the HeI $\mathrm{D}_{3}$ line exhibits emission in the observation of the optical band (the first one was reported by Montes et al. 1996). There is a significant trend for orbital phase modulation of chromospheric emission during our observing campaigns, and an activity longitude area was found around the second quadrature, where the mass from the secondary is transferring to the primary. Perhaps the chromospheric activity and mass transfer activity are affected by each other, and the coupling between the two kinds of activities makes the total system exhibit high activity around the quadratures.

Acknowledgements. The authors would like to thank the anonymous referee for his (her) suggestions and comments, which led to large improvement in the manuscript. $\mathrm{Gu}$ is very grateful to Dr. Montes for providing a copy of the STARMOD 


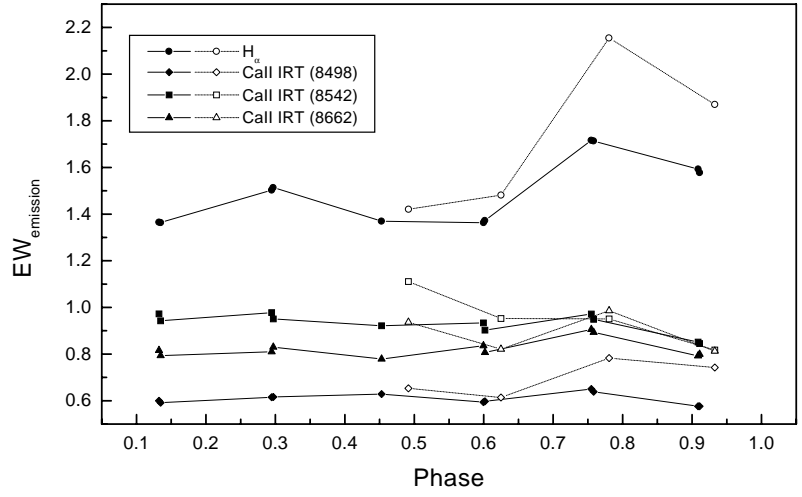

Fig. 8. The $E W$ of the excess emissions vs. orbital phase for $\mathrm{H} \alpha$ and CaII IRT lines. The solid symbols and open symbols represent the observations in Feb. and Sep.-Nov. 2000, respectively.

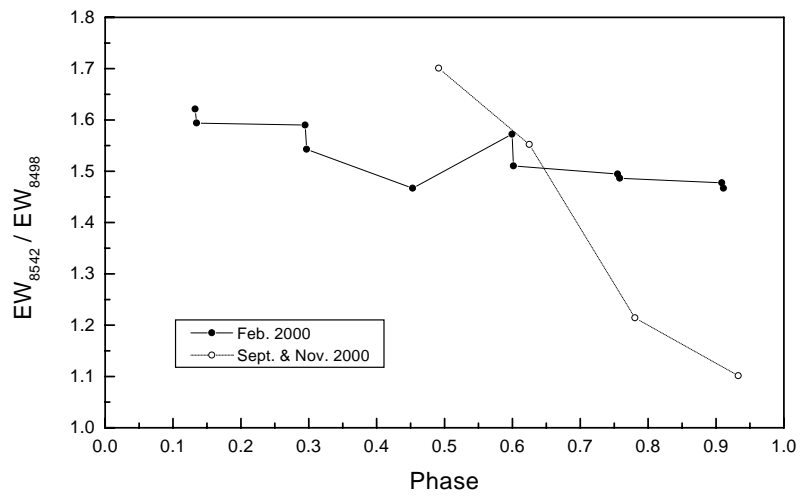

Fig. 9. The $E W_{8542} / E W_{8498}$ ratio vs. orbital phase. The solid symbols and open symbols represent the observations in Feb. and Sep.-Nov. 2000, respectively.

program. This work is supported by the Applied and Basic Research Foundation of Yunnan Province, China.

\section{References}

Arévalo, M. J., \& Lázaro, C. 1999, AJ, 118, 1015

Barden, S. C. 1985, ApJ, 295, 162

Carlos, R. C., \& Popper, D. M. 1971, PASP, 83, 504

Catalano, S., \& Frasca, A. 1994, A\&A, 287, 575

Duemmler, R., \& Aarum, V. 2001, A\&A, 370, 974

Foing, B. H., Char, S., Ayres, T., et al. 1994, A\&A, 292, 543

Frasca, A., \& Catalano, S. 1994, A\&A, 284, 883

Gunn, A. G., \& Doyle, J. G. 1997, A\&A, 318, 60

Gunn, A. G., Doyle, J. G., \& Houdebine, E. R. 1997, A\&A, 319,211

Hall, J. C. 1996, PASP, 108, 313

Hatzes, A. P. 1995, AJ, 109, 350

Huenemoerder, D. P., Buzasi, D. L., \& Ramsey, L. W. 1989, AJ, 98, 1398

Lázaro, C., \& Arévalo, M. J. 1997, AJ, 113, 2283

Montes, D., Fernández-Figueroa, M. J., De Castro, E., \& Cornide, M. 1995, A\&AS, 109, 135

Montes, D., Sanz-Forcada, J., Fernández-Figueroa, M. J., \& Lorente, R. 1996, A\&A, 310, L29

Montes, D., Fernández-Figueroa, M. J., De Castro, E., \& Sanz-Forcada, J. 1997, A\&AS, 125, 263

Montes, D., Fernández-Figueroa, M. J., De Castro, E., et al. 1998, in Cool stars, Stellar system, and the Sun 10th Cambridge workshop, ed. R. A. Donahue, \& J. A. Bookbinder, ASP Conf. Ser., 154, CD-1516

Montes, D., Fernández-Figueroa, M. J., De Castro, E., Cornide, M., Latorre, A., \& Sanz-Forcada, J. 2000, A\&AS, 146, 103 Nations, H. L., \& Ramsey, L. W. 1986, AJ, 92, 1403

Zirin, H. 1988, in Astrophysics of the Sun (Cambridge University Press) 